\title{
CONCEPTUALIZACIÓN, OBJETO DE ESTUDIO, EVOLUCION HISTORICA Y CUERPO DE CONOCIMIENTO DE LAS CIENCIAS DEL DEPORTE
}

\author{
Por: Álvaro José Gracia*
}

\section{INTRODUCCIÓN}

Las encuestas aplicadas en la Facultad de Educación Física de la Universidad Pedagógica Nacional, a los alumnos de últimos semestres demostraron que conceptualmente, los futuros Licenciados no tienen conocimiento de lo que significan las Ciencias del Deporte, ni poseen información sobre cuál es su objeto de estudio, estructura y evolución histórica. Este hecho sustentó y justificó la construcción del presente documento como lectura de apoyo básica dentro de la unidad de conceptualización de la asignatura de Pedagogía y Didáctica de la Educación Física.

A través de la Ciencia del Deporte se busca el mejoramiento y perfección de la naturaleza del hombre, de sus objetivos en el mundo y de las obligaciones que dependen de su misión. La Ciencia del Deporte es en su mayor parte ciencia de la educación, que posee amplia relación con el fenómeno del juego y muchas otras disciplinas como: filosofía, sicología, medicina, historia de la cultura, etnología y sociología, entre otras.

En nuestro medio hay insuficiente información que permita socializar lo que es Ciencia del Deporte en su estructura y características fundamentales, por tal razón, este documento pretende realizar una profundización en sus ámbitos esenciales para hacer conocer sus particularidades.

A través de la Ciencia del Deporte se busca formar profesionales que le den solución a los problemas del deporte; entrenamiento, juzgamiento, investigación, dirección administrativa, sistematización, planeación, recursos y, especialmente, que propendan por la formación de la cultura deportiva, así como de la actividad física, la utilización del tiempo libre y el fortalecimiento de la salud.

Durante el recorrido histórico del ser humano se ha podido apreciar la necesidad de preparación y programación adecuada de su estado físico, para poder cumplir con el ritmo de las exigentes actividades cotidianas; para la caza, la guerra, el trabajo y para los juegos populares, mientras en la actualidad se prepara para el deporte.

Para escribir sobre las Ciencias del Deporte, con un sentido profundo y riguroso, es obligatorio rememorar los conceptos que componen esta joven ciencia. Por esta razón se hace necesario delimitar su conceptualización, su objeto de estudio, su evolución histórica y su cuerpo de conocimiento (campos teóricos y campos temáticos).

\section{Ciencia}

La ciencia nace de la preocupación del hombre antiguo por saber y explicarse el mundo, es decir, conocer sus características para comprenderlo. La ciencia hace referencia a los conocimientos más evolucionados utilizados en la forma más adecuada para superar los conocimientos humanos a través de los cuales nuestras facultades intelectuales pretenden averiguar la naturaleza, cualidades y relaciones de las cosas y

\footnotetext{
* Profesor Facultad de Educación Física, U.P.N. 
entre las cosas. La ciencia es un sistema de conocimientos en desarrollo que se obtienen mediante los correspondientes métodos: empírico-analítico, historico-hermenéutico y critico-social, que se reflejan en conceptos exactos, cuya veracidad se prueba y demuestra a través de la práctica científica y social.

La ciencia es un sistema de conceptos acerca de los fenómenos y leyes del mundo externo y de la actividad espiritual de los individuos, que permite prever y transformar la realidad en beneficio de la sociedad. La ciencia es una forma de actividad humana históricamente establecida, una producción espiritual, cuyo contenido y resultado es la reunión de hechos orientados en un determinado sentido, de hipótesis y teorías elaboradas y de leyes que constituyen su fundamento, así como de procedimientos y métodos de investigación ${ }^{1}$.

Ciencia: del Lat. scientia, de scientis, p.a. de scire, saber, conocimiento cierto de las cosas por sus principios y causas. Discurso acerca de un campo de objetos determinado en el que, con un lenguaje sometido a ciertas reglas, se enuncian proposiciones susceptibles de demostración o comprobación. Cuerpo de doctrina metódicamente formado y ordenado, que constituye un ramo particular del saber humano, conocimiento o erudición ${ }^{2}$.

La ciencia es la tarea de relacionar leyes y teorías producto de la investigación, para formular predicciones confiables acerca de hechos todavía desconocidos. Esta afirmación orienta el sentido u objeto de la ciencia, como se planteó anteriormente, comprender y explicar los secretos de la vida y la naturaleza, haciendo un análisis lógico y coherente que está direccionado, siempre, a la búsqueda de mejorar la calidad de vida de todos los seres humanos, aunque algunos inventos e investigaciones no se estén aplicando adecuadamente en esta dirección.

La esencia del conocimiento científico consiste en la auténtica generalización de los hechos, razón por la cual, a través de lo casual descubre lo necesario, que se encuentra respaldado por leyes; tras lo singular, lo general y sobre esta base se lleva a cabo la previsión y prevención de diferentes fenómenos, objetos y acontecimientos. Con lo anterior se intenta dar fundamento a la gran tarea de las Ciencias del Deporte, teniendo en cuenta que su sentido vital es saber para prever y prever para actuar. Todo ello nace de las incógnitas e interrogantes que se han constituido en problemáticas del fenómeno deportivo.

\section{Deporte}

En la medida que el deporte se ha constituido en un inigualable fenómeno social, se ha hecho necesario el uso de la ciencia. Gracias a ello han nacido las Ciencias del Deporte. Pero entremos a definir que es deporte: según la Ley 181 del deporte de 1995, el deporte es en general, la específica forma de conducta humana caracterizada por una actitud lúdica y de afán competitivo, de comprobación o desafío, expresada mediante el ejercicio corporal y mental dentro de disciplinas y normas preestablecidas orientadas a generar valores morales, cívicos y espirituales ${ }^{3}$.

Pierre Parlebas (1991) plantea que la institucionalización es uno de los parámetros, que necesariamente debe ser tenido en cuenta para definir lo que es el deporte y poderlo

\footnotetext{
${ }^{1}$ KEDROV, M., B.,Spirrín, A. (1982). La ciencia, Méjico, Grijalbo S.A.

${ }^{2}$ DICCIONARIO ENCICLOPÉDICO SALVAT (1985). Barcelona, Salvat Editores. V. 7, pág. 29.

${ }^{3}$ Ley 182 del 18 de enero de 1995 . Por la cual se dictan disposiciones, para el fomento del deporte y se crea el sistema nacional del deporte. Título IV. Cap.1. Definiciones y clasificación. Pág. 12.
} 
diferenciar de otras actividades de tipo físico y competitivo que no pueden ser considerados como tal. Por eso, finalmente define deporte como una situación motriz, de competición, reglada e institucionalizada.

Hernández Moreno, José (1994) haciendo una síntesis de las características fundamentales referenciadas por varios autores (Cagigal, J, M., 1983 ${ }^{4}, 1984^{5}$ Cazorla L. M., $1979^{6}$ Magnane, G Parlebas, E), plantea que los rasgos esenciales, que nos permiten aproximarnos al concepto de deporte, se sitúan en los siguientes cinco aspectos: situación motriz, juegos, competición, reglas e institucionalización.

Etimológicamente, el término deporte es procedente del latín desportore, que significa distraerse. Pasó al francés como desport (descanso) y al inglés como sport (descanso, placer, diversión). En el siglo XX la palabra deporte se puede circunscribir a todas aquellas actividades físicas que buscan a través de la competencia, reglamentada y socialmente reconocida, mejorar un rendimiento y obtener el éxito. El deporte ha perdido mucho de sus características como juego y ha acentuado más el entrenamiento y la institucionalización (Parlebas Pierre, 1891).

Considerando que el deporte es un fenómeno social, resulta de vital importancia anotar que la competencia o agonística, que es característica esencial del deporte como producto de su profesionalización, en variados casos presenta comportamientos fuera de sus reglas morales, que provocan que la ética deportiva entre a enjuiciar, reflexionar y a catalogar esos hechos como malos (doping), y desde luego fuera del juego limpio, porque se busca a través de medios ilegales sacar ventajas para obtener victorias. La infracción también se da como respuesta a la exigencia inhumana de nuevas marcas, por parte de los entrenadores hacia los deportistas "deshumanizando el deporte", convirtiendo al ser humano en hombre máquina y exclusivamente productor de resultados. Esta concreta ejemplificación y otras de marcada complejidad son objeto de estudio de las Ciencias del Deporte.

\section{Ciencias del deporte}

Ciencia del deporte proviene del vocablo alemán Wissenshaft, que traduce ciencia en alemán, para el trabajo científico y sus resultados relacionados con un muy complejo sistema social llamado deporte. Este fenómeno está muy diversificado, tiene muchas facetas y tiene que ser visto a la luz de la ciencia, y la ciencia que trata con estos aspectos es llamada ciencia del deporte.

Hoy en día existen muchas ciencias y en el futuro existirán otras más, ya que el proceso de diferenciación es continuo. En este contexto tiene que ser establecido que la ciencia del deporte es una disciplina relativamente joven en el mundo de la ciencia, aún intentando conseguir un completo reconocimiento de la comunidad científica.

La Ciencia del Deporte es en gran parte ciencia de la educación; que tiene amplia relación con el fenómeno del juego y con muchas otras disciplinas como la filosofía, la sicología, la medicina, la historia de la cultura, la etnología y la sociología, entre otros.

Las Ciencias del Deporte son todas aquellas que tienen directa y especializada incidencia en el deporte; ellas se han agrupado e interrelacionado para darle una

\footnotetext{
${ }^{4}$ CAGigal, J., M. (1983). El cuerpo y el deporte en la sociedad. Revista Papers de Psicología N ${ }^{9} 20$. Barcelona Editorial Península.

${ }^{5}$ CAGIGAL, J., M. (1984). La pedagogía dello sporte come educacione. Revista de cultura Sportiva, No. 1, enero-marzo.

${ }^{6}$ CAZORLA, L, M. (1979). Deporte y estado. Barcelona, Editorial Labor 
estructura seria y ordenada, planteando material de trabajo para sí y para cada disciplina científica comprometida en esta tarea.

Las Ciencias del Deporte se conceptualizan como un sistema, y constituyen la interdisciplinariedad de ciencias especializadas que dirigen sus investigaciones con la intención de emplear los resultados en las aplicaciones técnicas del deporte, es decir, en la búsqueda del desarrollo adecuado de la actividad física, para el logro de la formación biopsicosocial del hombre y el mejoramiento de su rendimiento deportivo. (HAAG, Helbert, 1994).

Las Ciencias del Deporte comprenden un sistema relativamente nuevo y cambiante, dado el fenómeno típico de la corriente científica actual, en donde la rápida y rauda evolución de los conceptos y conocimientos científicos inciden directa y simultáneamente sobre las ciencias en mención, dando como resultado la aparición de nuevas disciplinas científicas.

En síntesis: Las Ciencias del Deporte representan un sistema de investigación científico, de enseñanza y práctica que necesita de la integración de otras disciplinas. (HAAG, Helbert, 1994).

\section{Objeto de estudio de las ciencias del deporte}

El objeto de estudio de las Ciencias del Deporte está integrado o constituido por todos aquellos interrogantes y preguntas que generen investigación científica en el deporte, porque han sido identificadas como problemas.

Las Ciencias del Deporte incrementan el conocimiento en función de las preguntas propuestas sobre una base científica. Finalmente propone soluciones y se aplica, con el fin de explicar, controlar y orientar cuando sea necesario, en la práctica del deporte.

Las Ciencias del Deporte son un ejemplo claro de lo que es integración, interdisciplinariedad, o campo científico de cruce disciplinar, en contraste con las antiguas ciencias establecidas como Filosofía, Medicina, Leyes y Matemáticas que han sido llamadas Ciencias orientadas disciplinarmete.

\section{Evolución histórica de las ciencias del deporte}

La historia de la Ciencia del Deporte hace su aparición hacia el año de 1900, precisamente en la época en que el deporte se va constituyendo como un fenómeno socio cultural de carácter mundial. Se convierte en una ciencia relativamente nueva y va encontrando su estructura a través de los primeros trabajos que buscan integrar algunas ciencias que trabajan o que dirigen sus investigaciones entorno al deporte como la Biología, la Filosofía y la Medicina, entre otros.

Hacia 1938 se elaboraron análisis más o menos completos sobre la importancia de las ciencias del Deporte, entre los que se destacó el trabajo de Karl Koch, en su libro "Hacia una ciencia del deporte" en el cual, se define las ciencia del deporte como "La ciencia del hombre en movimiento". Pero los constructos completos de su concreta estructura solo aparecen en el año de 1994 en Theoretical Foundation of Sport Science as a Scientific Discipline (Fundamentos Teóricos de la Ciencia del Deporte Como una Disciplina Científica) de Haag Herbert (1994). En donde se hace una presentación y análisis de lo 
ocurrido con las ciencias del deporte entre 1955 y 1985, y que corresponde a la forma como actualmente está fundamentada y se conoce las ciencias del deporte.

Los documentos elaborados entre 1955 y 1985 permiten visualizar, como se originó el proceso de desarrollo, dimensión y proyección histórica de las ciencias del deporte, mostrando tanto los objetivos y tareas como las estrategias y políticas mediante las cuales se implementó. Además, presentan los elementos fundamentales para su implementación y éxito, como son: la investigación, la promoción y la distribución de los resultados obtenidos en el campo de la Ciencia Deportiva, señalando que su aplicación es garantía para orientar los procesos de enseñanza - aprendizaje y rendimiento deportivo y finalmente, manifiesta que para el logro de lo anterior habrá cooperación Nacional e Internacional.

A continuación se hace mención y descripción de los principales documentos (estatutos y decretos, entre otros) escritos sobre esta temática en busca de su desarrollo entre los años 1955 y 1985.

En 1955 el Consejo Para la Investigación de la Medicina Deportiva (Kuratorium Fur die sportmedizinische Forschung), emitió el siguiente estatuto: "El Consejo Para la Investigación de la Medicina Deportiva fundado por la Federación Deportiva Alemana, tiene como tarea, realizar investigaciones acerca de Medicina Deportiva, con el fin de servirle a la gente, en particular a la juventud. Por eso, deberá atender toda inquietud concerniente en este campo, siendo responsable de sintetizar los resultados, coordinar y guiar investigaciones futuras".

En 1964 se fundó el Comité Central para la Investigación en el Deporte, que adoptó, entre otros, los siguientes compromisos:

- Dar respuesta a todos los problemas que tienen que ver con la investigación de la ciencia deportiva, observando su importancia fundamental en la Educación Física.

- Incentivar, coordinar y controlar todo proyecto investigativo que se lleve a cabo, teniendo en cuenta, todas las tareas puestas en marcha desde un criterio científico.

- Permitir la intervención investigativa en las diferentes áreas teóricas por parte de investigadores reconocidos por sus labores científicas, la cual es necesaria especialmente para ayudar a la gente.

- Facilitar las condiciones para la difusión de los últimos resultados provenientes de la investigación de la ciencia deportiva.

Aunque, aquí, no hay conceptos muy claros, sí se puede determinar la riqueza de información que existe al analizar la dimensión histórica. El marco referencial de la evolución histórica sirve para no desperdiciar esfuerzos al realizar investigaciones de temas ya resueltos (Grupe 1968, 1971).

\section{En 1966 se decretó “La Carta del Deporte Alemán” la cual dice:}

"Tanto la educación física como el deporte le dan a la ciencia nuevas tareas, los institutos de aprendizaje superior también toman decisiones de cual va a ser su rumbo en el futuro y cual es su status en la sociedad actual. Solo con los institutos de educación superior sería posible mejorar las ideas prejuiciosas de la educación. Para que se pudiese 
lograr esa meta fue necesario: asegurar una posición equitativa con respecto al objeto de estudio y evaluación en comparación con las demás materias que regulan el objeto de estudio y la evaluación de futuros educadores físicos; asegurar la investigación y la enseñanza del deporte, de la educación física por medio de la creación de carreras en ciencia deportiva; agrandar los institutos de educación física dentro de los institutos de educación superior".

En 1976 se funda la Asociación Alemana para las Ciencias del Deporte (Dents Che Vereinigung fur Sport Wissens Chaft) (DVS): que tiene como propósito promover el futuro desarrollo de la ciencia del deporte. Sus tareas eran específicamente:

- Generar y apoyar la investigación científico deportiva.

- Iniciar y organizar la comunicación científica entre y dentro de los campos teóricos de la ciencia deportiva.

- Determinar los objetos de estudio y aprendizaje en la ciencia deportiva.

- Apoyar el desarrollo de la estructura de los diferentes institutos educativos de ciencia deportiva.

- Generar el surgimiento de científicos deportistas jóvenes.

En 1978 la UNESCO adoptó la carta para la educación física y el deporte, cuyo artículo sexto hace referencia a la Ciencia Deportiva. "La investigación y la evaluación son partes necesarias en el desarrollo de estas áreas".

- Tanto la investigación como la evaluación deben promover el mejoramiento de todas las formas del deporte y contribuir a mejorar la salud y la seguridad de quienes lo practican, de igual manera, fortalecer y estructurar los métodos de entrenamiento y los métodos organizativos y administrativos. La educación se vería beneficiada porque se podrían propiciar nuevos y mejores métodos educativos con un mejor nivel de rendimiento.

- La investigación científica y la influencia social no se pueden pasar por alto.

En 1983 en el consejo Internacional Para la Ciencia Deportiva y la Educación Física, adoptó estatutos con pronunciamientos referentes a la ciencia deportiva.

- El Consejo Internacional Para la Investigación Científica en Deporte y Educación Física (ICSSPE) es una organización interesada en la promoción y distribución de los resultados obtenidos en el campo de la ciencia deportiva y su aplicación en contextos culturales y educativos.

- Los objetivos fundamentales de la ICSSPE serán: Impulsar la cooperación internacional en el campo de la ciencia deportiva para beneficio de todos: promover, estimular y coordinar la investigación científica en el campo de la ciencia deportiva y la educación física en el mundo entero, además de apoyar la aplicación de sus resultados en diferentes áreas prácticas del deporte; poner a disposición de toda organización deportiva nacional e internacional todo conocimiento científico y experiencia práctica deportiva; iniciar, coordinar y organizar conferencias internacionales, simposios, reuniones, seminarios y clínicas; incitar y proveer la publicación del conocimiento en ciencia deportiva y estimular la socialización de la idea y los resultados, por parte de los 
medios; cooperar con las organizaciones nacionales e internacionales en el campo de la educación física, la ciencia deportiva y las demás ciencias.

\section{Cuerpo de conocimiento de las ciencias del deporte}

El modelo para describir el cuerpo del conocimiento de las Ciencias del Deporte intenta responder este nuevo reto del campo del deporte en relación con su percepción científica. Las distinciones necesarias que se dan dentro del cuerpo de conocimiento de las Ciencias del Deporte son las siguientes: Campos Teóricos y Campos Temáticos.

El Campo Teórico se refiere a una subdisciplina aplicada de una disciplina académica más o menos establecida con una historia relativamente antigua. Esta es llamada Intra relación entre la subdisciplina aplicada y la madre - académica o ciencia madre. Es decir, que la teoría es más abstracta y tiene un carácter orientado hacia una disciplina. (HAAG Helbert, 1994)

El Campo Temático o de temas es considerado como unidad pues está compuesto de interrelaciones entre diferentes campos teóricos relacionados con ciertos temas, que pueden venir directamente del campo de movimiento, el juego y el deporte, o que podrían ser un tema con dimensiones que no son solo específicas del deporte. Los temas se representan de una manera directa y concreta.

\section{Clasificación de los campos teórico establecidos por las ciencias del deporte.}

- Medicina del deporte.

- Sociología del deporte.

- Biomecánica del movimiento en el deporte.

- Historia del deporte.

- Psicología del deporte.

- Filosofía del deporte.

- Pedagogía del deporte.

\section{Clasificación de los nuevos campos de teoría de la ciencia del deporte}

- Información deportiva: Ciencia de la información y deporte.

- Política deportiva: Ciencia política y deporte.

- Legislación deportiva: Ciencias legales y deporte.

- Teoría de instalaciones y equipos deportivos:

Ciencias técnicas orientadas y deportes-

- Economía deportiva: Economía y deporte.

\section{Campos temáticos específicos del deporte en las ciencias del deporte}

- Ciencia del movimiento: Teoría del movimiento.

- Ciencia del juego: Teoría del juego.

- Ciencia del entrenamiento: Teoría del entrenamiento.

- Ciencia de la instrucción: Teoría de la Institución.

\section{Campos temáticos generales de las ciencias del deporte}

- Aplicación y capacidad de ejecución en el deporte.

- Música y movimiento. 
- Deporte y recreación.

- Deporte y salud.

- Deporte con grupos especiales.

- Deporte y medios masivos.

- Agresión y violencia.

Actualmente, las Ciencias del Deporte siguen su evolución y desarrollo en la búsqueda del fortalecimiento de su identidad, estructura, terminología y organización, mediante su constante trabajo científico, dirigido a mejorar los problemas y dificultades encontrados dentro del fenómeno deportivo actual.

\section{Conclusión}

El presente documento ha mostrado el desarrollo, dimensión y evolución histórica de las Ciencias del Deporte, señalando cómo en cada paso tuvo y sigue teniendo objetivos y tareas claras, estrategias y políticas operativas, que le han permitido lograr su implementación científica.

Hay elementos que han sido estrategias esenciales para la estructuración y fortalecimiento de las Ciencias del Deporte, de tal manera que es la investigación sobre los problemas que se presentan en el deporte y en la actividad física, la que junto a la promoción y distribución de los resultados obtenidos en el campo de la ciencia deportiva, la que ha permitido ir consolidando y fortaleciendo las Ciencias del Deporte.

La Ciencia del Deporte es en gran parte Ciencia de la Educación, principalmente visible a través de su cuerpo de conocimiento, integrado por el Campo Teórico y el Campo Temático. Ha surgido como resultado de un gran esfuerzo científico en relación con el Deporte, conformando un sistema de integración interdisciplinaria o campo científico de cruce disciplinar de ciencias especializadas que dirigen sus resultados hacia la formación biopsicosocial del deportista, bajo la filosofía: "Primero los deportistas y segundo ganar" (Thompson Pete; 1991), perfeccionando en esta forma la actitud y aptitud del ser humano ante el deporte y la vida, sobre la base de principios y leyes generales que constituyen ese conjunto de conocimientos unidos, que apuntan hacia un objeto de estudio común, la investigación y la solución de los problemas y / o situaciones deportivas encontradas, lo que le da la categoría de ciencia.

\section{Bibliografía}

CAGIGAL, J.M. (1983). El cuerpo y el deporte en la sociedad. Revista Papers de sociología $N^{\circ}$. 20. Barcelona: Editorial Península.

CAGIGAL, J.M. (1984). La pedagogía dello sporte come educaciones, revista de cultura sportiva, No. 1, Enero - Marzo, 1984.

CAZORLA, L. M. (1985). Deporte y estado. Barcelona: Salvat Editores. V. 7. Pág 29.

DICCIONARIO ENCICLOPÉDICO SALVAT. Barcelona: Salvat Editores. V. 7. 1985. Pág. 29

LEY 182 DEL 18 DE ENERO DE 1995. Por la cual se dictan disposiciones, para el fomento del deporte y se crea el sistema nacional del deporte. TíTULO IV. Capítulo I. Definiciones y Clasificación. Pág 12. 
HAAG, Herbert, (1994). Theoretical Eoundation of Sport science as a scientific discipline. Primera edición. Federación de la República de Alemania.

HERNÁNDEZ MORENO, José. (1994). La delimitación del concepto deporte y su agonismo en la sociedad de nuestro tiempo. Armenia (Quindío): Editorial Kinesis. $\mathrm{N}^{9} 13$. Pág. 23.

HOFMANN, Karl. (1994). Contribution to a philosophy of sport science, Germany.

LEY 181 del 18 de Enero de 1995. Por la cual se dictan disposiciones para el fomento del deporte y se crea el Sistema Nacional del deporte Título IV. Capítulo I. Definiciones y clasificación, pág 12.

KÉDROV, M.B. Y SPIRKIN, A. (1982). La ciencia, México: grijalbo, S.A., Pág. 7.

KOCH, Karl. (1981). Hacía una ciencia del Deporte. Buenos Aires: Editorial Kapelusz S.A.

THOMPSON, Pete; (1991). Introducción a la teoría del entrenamiento, Londres: Internacional Amateur Athetic Federation. Pág 1-5. 